\title{
Desenho de Produto de Tecnologia Assistiva Considerando as Pessoas, o Contexto de uso e as Atividades
}

\author{
Soraia Silva Prietch, Polianna dos Santos Paim, Nivaldo Alexandre de Freitas, \\ Waine Teixeira Junior \\ Universidade Federal de Rondonópolis - Av. dos Estudantes, 5055 - 78.736-901 - \\ Rondonópolis - MT - Brasil \\ \{soraia, waine\}@ufr.edu.br, \{nivafreitas, poliannapaim\}@gmail.com
}

\begin{abstract}
Assistive Technology (AT) products can promote more equality in opportunities, quality of life and autonomy to people with disabilities. To design these products and improve their adoption by users, four main components must be taken into account: the Human, the Activity, the AT, and the Context. In this paper, we describe the path taken in a research project with the goal to study the performance of diverse activities by people with disabilities in different contexts of use. In five years of the research project, we conducted eight projects with specific themes and objectives. We can highlight the importance of participatory activities with the community, more than the target-users, e the inclusion of people with disabilities in the project leadership.
\end{abstract}

Resumo. Produtos de Tecnologia Assistiva (TA) podem promover para pessoas com deficiência maior igualdade nas oportunidades, qualidade de vida e autonomia. Para o desenho destes produtos e para potencializar a sua adoção, é preciso que quatro componentes essenciais sejam considerados: Humanos, Atividades, TA e Contexto de uso. Neste artigo é descrito o caminho percorrido em um projeto de pesquisa cujo objetivo foi investigar a realização de variadas atividades por pessoas com deficiência em diversos contextos de uso. Durante os cinco anos do projeto, oito projetos que contavam com temas e objetivos mais específicos foram conduzidos. $E^{\prime}$ possivel destacar a importância de atividades participativas com a comunidade, para além do público-alvo, e a inclusão de pessoas com deficiência na liderança de projetos.

\section{Introdução}

Este artigo relata brevemente sobre uma coletânea de projetos de pesquisa que é parte integrante do projeto maior formado por pesquisadores de Mato Grosso (Universidade Federal de Rondonópolis (UFR), Universidade Federal de Mato Grosso (UFMT) e Universidade do Estado de Mato Grosso (UNEMAT)/ Câmpus de Sinop) e de Minas Gerais (UFLA) - que se reuniram em torno de tema de interesse comum, durante cinco anos. Vale ressaltar que o projeto maior foi analisado pelo Comitê de Ética em Pesquisas com Seres Humanos antes de iniciar suas ações.

A ideia central desse projeto maior era a de realizar os mesmos procedimentos de investigação em diferentes instituições, localizadas em diferentes cidades, com o público 
sendo pessoas com deficiência (ex., pessoas surdas, pessoas cegas, pessoas com autismo, etc.) em diversos contextos de uso (ex., escola, reabilitação, universidade, etc.) e necessidades de realização de variadas atividades (ex., leitura, escrita, socialização, etc.) com o intuito de desenhar, desenvolver e avaliar produtos de Tecnologia Assistiva $(\mathrm{TA})^{1}$. Esse modo de refletir questões relacionadas às barreiras de acessibilidade e de realizar a pesquisa foi suportado pelo modelo HAAT (Human Activity Assistive Technology), de Cook e Polgar (2008), o qual prevê o desenho de produtos de TA considerando não somente a tecnologia ou a pessoa com deficiência isoladamente, mas os ambientes em que o produto será essencial para aquela pessoa realizar atividades específicas. Sendo assim, o objetivo deste artigo é descrever sobre o caminho percorrido por uma das instituições envolvidas (UFR), relatando as atividades de investigação realizadas e os resultados obtidos neste percurso.

O restante do artigo está organizado da seguinte maneira: na Seção 2, apresentamos materiais e métodos utilizados para realizar a coletânea de projetos de pesquisa locais. A Seção 3 está subdividida para relatar sobre os resultados por projeto e, na Seção 4, descrevemos as considerações finais.

\section{Materiais e métodos}

Apoiados no modelo HAAT, a intenção ao longo do projeto local foi a de conhecer e envolver pessoas com deficiência e pessoas que possuem contato direto com elas, de modo a envolver as partes interessadas no desenho de produtos de tecnologia assistiva, com considerando os ambientes e as interações sociais. Neste projeto, diversos subprojetos foram executados com objetivos mais específicos e metodologias próprias para a sua realização. Portanto, realizamos os oito projetos, aqui organizados por temas, tendo forte engajamento de estudantes do curso de graduação em Sistemas de Informação da UFR em iniciação científica, em projeto final de curso ou em estágio.

A Figura 1 apresenta a linha do tempo destes cinco anos de realização de subprojetos relacionados ao objetivo central dos projetos local e global. Portanto, em 2017 e 2018 continuamos dois temas que se encontravam em andamento: "Instrumento inclusivo para o auto relato da experiência emocional" e "Sistema colaborativo para a divulgação de tecnologia assistiva". Entre 2017 e 2019, realizamos atividades investigativas com os temas "Uso de TA na escola" e "Suporte para a leitura e a escrita de pessoas usuárias da Libras". Em 2020, devido à pandemia, nos interessamos em conhecer mais sobre os desafios e soluções alternativas que os professores vivenciavam no contexto da educação inclusiva. De 2019 a 2021 formamos parcerias com pesquisadores do México e desenvolvemos investigações sobre questões socioculturais para o desenho de sistemas de Processamento Automático de/para Língua de Sinais (PALS). Ao longo deste período, houve o envolvimento de pessoas surdas usuárias da Língua Brasileira de Sinais (Libras), intérpretes, professores de escolas públicas e de educadores de diferentes áreas de conhecimento: Computação, Psicologia e Letras. Esse envolvimento evidencia que este tipo de estudo é interdisciplinar e se faz necessário conhecer os diversos círculos

\footnotetext{
${ }^{1}$ Neste artigo, utilizamos a definição de TA conforme Brasil (2015, Art. $3^{\circ}$ ), a qual se refere a uma área de conhecimento e que envolve a proposição e a aplicação de produtos, serviços, estratégias, dentre outros, para promover as mesmas condições de oportunidades das pessoas com deficiência comparada às pessoas sem deficiência, além de conferir suporte à qualidade de vida com maior autonomia.
} 
sociais que influenciam e são influenciados pelas pessoas a quem desejamos beneficiar com os frutos da pesquisa sobre tecnologia.

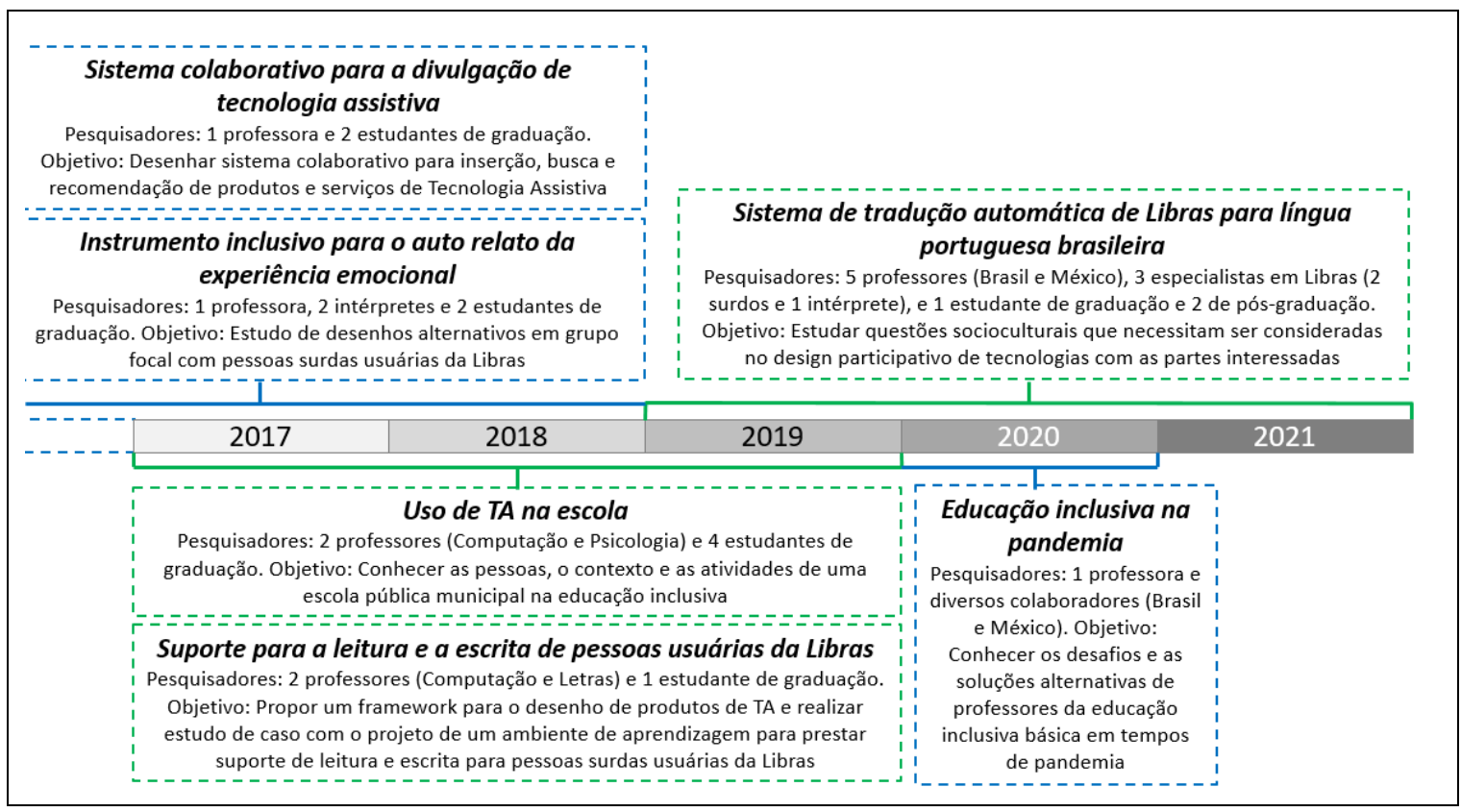

Figura 1. Linha do tempo e os temas dos subprojetos

Como o projeto maior é financiado por órgão de fomento, este teve início e fim determinados e redefinidos, devido à pandemia, em edital e determinações em diário oficial. No entanto, o projeto PALS se integra a outro em andamento, o qual tem vigência até 2022 e tem aberto outras oportunidades de parcerias no México.

\section{Resultados}

Nesta seção, descrevemos em linhas gerais os resultados obtidos em cada subprojeto, sendo que na Figura 2 apresentamos algumas fotos registradas nestes.

(a)

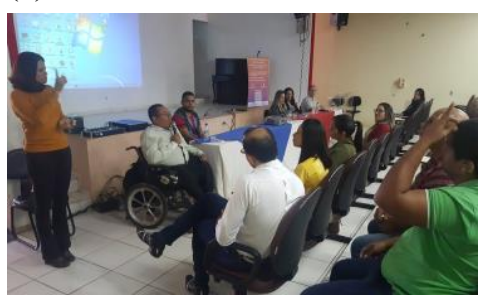

(b)

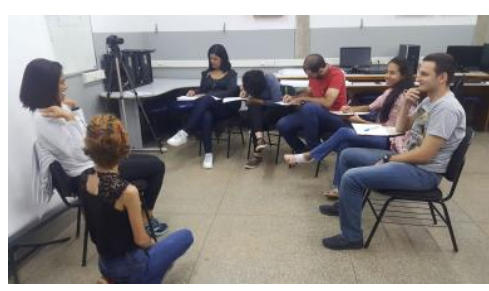

(c)

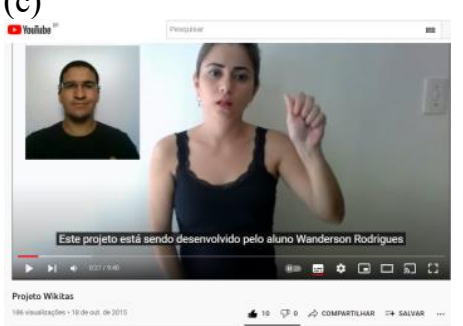

Figura 2. Fotos do (a) Encontro de Inclusão Social e Políticas Públicas; (b) Projeto de suporte à leitura e a escrita para Surdos; (c) Projeto Wikitas ${ }^{2}$

\subsection{Instrumento inclusivo para o auto relato da experiência emocional}

Este é um instrumento que vem sendo trabalhado há mais tempo e durante o presente projeto, foram desenvolvidos três protótipos e levamos para avaliação em grupo focal

\footnotetext{
${ }^{2}$ Apresentação na $1^{\mathrm{a}}$ FECITI - Feira Municipal de Ciência e Tecnologia e Inovação de Rondonópolis, 2015. Disponível em: https://www.youtube.com/watch?v=kdCng92ksRI, acesso dia 07 Nov. 2021.
} 
com pessoas surdas usuárias da Libras. Tal instrumento conta com vídeos em Libras, português escrito e falado, e o público-alvo são pesquisadores que desejam avaliar a experiência emocional dos usuários após interagir com tecnologias. Realizamos uma sessão de grupo focal na associação de surdos local, a qual contamos com a participação de cinco pessoas surdas usuárias da Libras. $O$ objetivo do estudo foi conhecer as percepções dos participantes a respeito dos três protótipos desenhados para $\mathrm{o}$ instrumento. Mesmo dada a preferência dos participantes pelo Protótipo 2, durante a sessão, identificamos quatro lacunas potenciais para melhorias: qualidade dos vídeos, escolha da língua, rótulos escritos e controle do usuário. Outro aprendizado foi a importância de incluir a liderança de uma pessoa surda no grupo de pesquisa. Os resultados desta pesquisa estão em processo de publicação [Prietch et al. (2021)] e, pretendemos disponibilizá-lo para uso pela comunidade geral.

\subsection{Sistema colaborativo para a divulgação de tecnologia assistiva}

A motivação desta pesquisa é que não existe uma ferramenta, chamada Wikitas (Figura 2c), que converge informações sobre tecnologias assistivas no Brasil. Muitas vezes as escolas recebem recursos para aquisição de produtos de TA e precisam passar muitas horas consultando diferentes websites à procura de ideias para auxiliar seus estudantes. Um sistema colaborativo permite que as pessoas possam tanto consultar quanto incluir informações sobre nomes, onde comprar e também relatar experiências que tiveram com o uso daquele produto ou serviço de TA. Este sistema web contou com a colaboração de dois estudantes em anos consecutivos, os quais o modelaram e o desenvolveram. Avaliações foram realizadas por potenciais usuários sendo que melhorias na interface e na oferta de funcionalidades foram promovidas. O trabalho foi publicado [Silva e Prietch, 2016] e, atualmente, com recursos de órgão de fomento pretendemos disponibilizá-lo para uso pela comunidade geral.

\subsection{Uso de TA na escola}

Em diálogo com a gerência de educação inclusiva da Secretaria Municipal de Educação, uma escola foi definida para a realização de pesquisa em campo. A escola se situa em bairro periférico, mas era considerada um exemplo de escola acessível. Após reuniões com a comunidade escolar, entre 2018 e 2020, estivemos na escola majoritariamente efetuando reconhecimento do ambiente, das pessoas, das atividades e do uso de tecnologias. Realizamos entrevistas com 1 gestor, 3 professores de sala de aula regular, e 2 professores da sala de recursos para nos familiarizarmos com as pessoas no contexto. O roteiro incluía perguntas sobre acessibilidade comunicacional, metodológica, programática e atitudinal [Manzini (2008); Sassaki (2009); Kleina (2012)]. Em um segundo momento, efetuamos inspeção minuciosa de acessibilidade espacial [Dischinger et al. (2009)] usando a lista de verificação com 18 seções com, em média, 4 perguntas cada. Foi interessante conhecer todos os espaços da escola, no entanto, consideramos ao final que teria sido mais interessante efetuar a inspeção com lista de verificação dos produtos de TA disponíveis. Elaboramos esta segunda lista adaptada de Manzini et al. (2008) e Manzini (2013) - questionários sobre Tecnologia Assistiva para Educação (TAE I e TAE II) -; no entanto, esta inspeção não foi realizada. Por fim, realizamos observações do AEE com 14 estudantes com diversos tipos de deficiência, atendidos nos períodos matutino e vespertino. Essa atividade teve duração de, em torno de seis meses. 
Em paralelo planejamos o uso de um diário de experiência do usuário e a seleção de TA, no entanto, a escola não aceitou mais participar do projeto. Esta pesquisa ainda não foi publicada, mas realizamos o evento "I Encontro de Inclusão Social e Políticas Públicas: Inclusão escolar e tecnologias inclusivas para PcD”3 (Figura 2a) na UFR em 2018.

\subsection{Suporte para a leitura e a escrita para pessoas usuárias da Libras}

Efetuamos a proposta de um framework para o desenho de produtos de TA construído com base nas teorias de Design Socialmente Consciente, Design de Interação e Framework para a adoção de TA. Este framework é formado por quatro etapas para desenho de produtos de TA: (1) Explorar e Entender, (2) Desenhar e Desenvolver, (3) Avaliar e (4) Monitorar. Como forma de validação do framework proposto foi realizado estudo de caso com o projeto de um ambiente de aprendizagem para prestar suporte de leitura e escrita para pessoas surdas usuárias da Língua Brasileira de Sinais (Libras). O suporte teórico serviu tanto para organizar as etapas quanto para definir as 17 atividades de investigação realizadas, nas quais trinta pessoas voluntárias da comunidade geral foram envolvidas (Figura 2b) e 4 artigos foram publicados [Paim, Prietch e Duarte (2018); Paim e Prietch (2019a) e (2019b); Paim, Prietch e Sánchez (2021)] ao longo dos anos em que esta pesquisa foi executada. Esta foi a pesquisa que mais envolvemos codesigners e nos proporcionou perceber a importância do lema "nada sobre nós, sem nós" no projeto de TA para pessoas com deficiência.

\subsection{Sistema de tradução automática de Libras para língua portuguesa brasileira}

Este projeto contou com revisão de literatura inicial e com alguns testes para o reconhecimento de alguns gestos. As produções dos estudantes envolvidos foram resumos em seminários de iniciação científica local e, a partir desta proposta, também conquistamos publicações com colaboradores internacionais [Silva-Prietch et al. (2019)]. Essa pesquisa tomou proporção maior após as publicações, a qual nos levou a propor novo projeto de pesquisa especializado nesta linha de estudos.

\subsection{Educação inclusiva na pandemia}

Um questionário online foi aplicado em 2020. O convite aos professores da educação básica foi divulgado pelas gerências de educação inclusiva das Secretarias Municipais de Educação das duas cidades investigadas: Rondonópolis, Mato Grosso - Brasil, e Puebla, Puebla - México. O questionário continha duas partes: 5 perguntas fechadas sobre dados demográficos e 8 perguntas abertas na forma de relato de experiências (contexto, legislação, caracterização do público atendido, uso de tecnologias, desafios, participação dos pais, materiais didáticos de terceiros e materiais didáticos de própria autoria). No total obtivemos 80 respostas, sendo 20 da cidade brasileira e 60 da cidade mexicana. Foi possível identificar mais semelhanças do que diferenças, tanto nos dados demográficos quanto nas práticas pedagógicas com uso de tecnologia durante a pandemia. Esta pesquisa ainda não foi publicada, mas foi apresentada em evento da Benemérita Universidad Autónoma de Puebla (BUAP), México.

\footnotetext{
${ }^{3}$ Chamada para participação do Encontro em Libras. Disponível em:

https://www.youtube.com/watch?v=wppRmK7uJEs\&t=20s, acesso dia 05 Nov. 2021.
} 


\section{Considerações finais}

Este projeto consistiu em uma longa jornada de produção de conhecimento, de formação de estudantes para suas potenciais carreiras como pesquisadores e fortalecimento de laços com as instituições parceiras para a realização de outros e novos projetos colaborativos. Dentre os temas citados, é possível afirmar que três se destacaram (subseções 3.1, 3.2, 3.4 e 3.5), tanto pela profundidade atingida nos estudos, quanto pela produção de artigos relevantes para o avanço da área de Interação Humano-Computador e de acessibilidade, e pelo potencial de geração de registro de software.

O conjunto dos projetos, porém, permitiu a produção de conhecimento compatível com os objetivos da pesquisa, no sentido da obtenção de elementos que, ao conferir maior autonomia aos sujeitos, permitem aumentar a qualidade de vida de pessoas com deficiência. Além disso, os produtos de tecnologia assistiva descritos nas subseções possuem a potencialidade de promover condições de igualdade das pessoas com deficiência em relação às pessoas sem deficiência. Tal potencialidade obtida nos produtos da pesquisa é de valor social e político indescritível ao se pensar em inclusão social. Para os resultados obtidos foi essencial também a consideração dos ambientes em que determinada tecnologia seria empregada, indo além da pesquisa concentrada apenas no usuário ou na tecnologia. É certo que as avaliações realizadas permitem avançar no aprimoramento das tecnologias pelo fato de desvelarem tanto lacunas e imprecisões, como acertos e potencialidades a serem desenvolvidas em cada produto especificamente.

Cabe destacar o conhecimento produzido ao longo da pesquisa no processo de interação com a pessoa com deficiência, que enriquece a compreensão dos produtos de tecnologia assistiva. Em primeiro lugar, pesquisas sobre inclusão e suas tecnologias não prescindem da relação com os agentes ligados à pessoa com deficiência, como família e comunidade, evidenciando que não basta transformar o interior da escola ao se objetivar uma sociedade inclusiva, sendo necessário também que a sociedade se faça presente na escola para o desenvolvimento do modelo inclusivo. Em segundo lugar, a pessoa com deficiência assumindo protagonismo na pesquisa de forma a liderar parte do projeto é algo fundamental e coerente com o que se espera de uma pesquisa que tem a inclusão como meta de forma geral, e a avaliação do uso de TA de forma específica.

\section{Agradecimentos}

Agradecemos o suporte financeiro da Fundação de Amparo à Pesquisa do Estado de Mato Grosso (FAPEMAT), Edital de Redes 037/2016.

\section{Referências}

Brasil. (2015). Lei n ${ }^{\circ}$ 13.146, de 6 de Julho de 2015. Institui a Lei Brasileira de Inclusão da Pessoa com Deficiência (Estatuto da Pessoa com Deficiência). Disponível em: t.ly/w97A. Acesso em: 25 set. 2016.

Cook, A. M. e Polgar, J. M. (2008). Cook and Husseys's Assistive Technologies: Principles and Practice. $3^{\mathrm{a}}$ ed., Elsevier.

Dischinger, M., Ely, V. H. M. B., Borges, M. M. F. da C. (2009). Manual de acessiblidade espacial para escolas: o direito à escola acessível. Brasília : Ministério da Educação, Secretaria de Educação Especial, 2009. 115 p. 
Kleina, C. (2012). Tecnologia assistiva em educação especial e educação inclusiva. Curitiba: InterSaberes.

Manzini, E. J. (2008). Tecnologia assistiva: definição, descrição e aplicação. Revista Brasileira de Educação Especial. Associação Brasileira de Pesquisadores em Educação Especial - ABPEE, v. 14, n. 3, p. 511-512. Disponível em: https://repositorio.unesp.br/handle/11449/30116. Acesso em: 16 mai. 2018.

Manzini, E. J., Maia; S. R. e Gasparetto; M. E. R. F. (2008). Questionário T.A.E. Tecnologia Assistiva para Educação. Brasília: Comitê de Ajudas Técnicas.

Manzini, E. J. (2013). Questionário TAE II: tecnologia assistiva para educação. Rio de Janeiro: UERJ, $11 \mathrm{p}$.

Paim, P., Prietch, S. e Duarte, A. (2018). CoDesign in the Exploratory Phase of an Assistive Technology product Design to support the Teaching-Learning Process of Brazilian-Portuguese Language for Visual Persons. In Proceedings of the 17th Brazilian Symposium on Human Factors in Computing Systems (IHC 2018). ACM, Article 12, 1-9. DOI:https://doi.org/10.1145/3274192.3274204

Paim, P. e Prietch, S. (2019a). Semiotic ladder artifact to design an assistive technology product for people who are deaf. In Proceedings of the IX Latin American Conference on Human Computer Interaction (CLIHC '19). Association for Computing Machinery, New York, NY, USA, Article 21, 1-5. DOI:https://doi.org/10.1145/3358961.3358982

Paim, P. e Prietch, S. (2019b). Communicability evaluation of video-exam in libras of the ENEM platform. In Proceedings of the 18th Brazilian Symposium on Human Factors in Computing Systems (IHC '19). Association for Computing Machinery, New York, NY, USA, Article 19, 1-11. DOI:https://doi.org/10.1145/3357155.3358478

Paim, P., Prietch, S. e Sánchez, J. A. (2021). Co-designing a Learning Environment for Written Representations of a Second Language for and with D/deaf Learners. In Proceedings of the 8th Mexican Conference on Human-Computer Interaction (MexIHC 2021). December 1--3, 2021, Online, México.

Prietch, S. S., Miranda, F., Paim, P., Leite, V., Soncini, C. (2021). A Sign Language Self-Report Instrument for Research on Emotional User Experience. In Proceedings of the X Latin American Conference on Human Computer Interaction (CLIHC 2021).

Sassaki, R. K. (2009). Inclusão: acessibilidade no lazer, trabalho e educação. Revista Nacional de Reabilitação (Reação), São Paulo, Ano XII, mar./abr., p. 10-16.

Silva, W. R. da e Prietch, S. S. (2016). Wikitas: Online Collaboration concerning with Assistive Technologies for Teachers of Students who are Deaf. In Proceedings of the 15th Brazilian Symposium on Human Factors in Computing Systems (IHC '16). ACM, Article 41, 1-4. DOI:https://doi.org/10.1145/3033701.3033743

Silva-Prietch, S., Gonzalez-Calleros, J. M., Sánchez, J. A., Olmos-Pineda, I., GuerreroGarcía, J. (2019). Cultural aspects in the user experience design of an ASLR system. In: CLIHC '19: IX Latin American Conference on Human Computer Interaction, 2019, Panama City Panama. Proceedings of the IX Latin American Conference on Human Computer Interaction. New York: ACM, 2019. p. 1-5. 